\title{
Source Localization by Conjugated Delays Method Main Description of a New Process of Passive Localization
}

\author{
Philippe Crumeyrolle
}

Umechanics, Umechanics R\&D, 7500, Paris, France

\begin{abstract}
The method of the combined delays is a passive tracking process. It is a triangulation method employed to locate the signal hearth, which can be, according to the application area, of acoustic or electromagnetic origin. This method is based on the process of the delays combination, (delays which result from the differences in distances between the source and the sensors of the reception station), which makes it possible to determine emission direction. By knowledge of angles measurements of the signal arrivals on the reception sensors of station, one completely determines the triangles, which establish the source position.
\end{abstract}

Keywords Localization, Passive, Triangulation, Delay

\section{Introduction}

The purpose of goniometry is to estimate wave incidence angles. These waves may be electromagnetic or acoustic. Goniometers are made up of an antenna or network of antennas comprising several sensors configured in a given geometry, and a calculation algorith $\mathrm{m}$. A radiogoniometer is a system capable of measuring the arrival direction of an electro magnetic wave with respect to a reference direction. The radiogoniometer samples an interpretation of the wave front of the electro magnetic wave over a network of several sensors to determine the arrival direction. The acoustic goniometer is a system that measures the arrival direction of sounds and thus estimates the direction of the emission source. Like the radiogoniometer, it is made up of an antenna configured in a geometry corresponding to the acoustic domain and a calculation algorithm. The localisation of sound sources by acoustic goniometry is based on a space-time process implemented in two steps. The temporal step extracts the arrival time of the signal or the differences in propagation times at the sensors of an antenna. The second step determines the direction of arrival according to the spatial configuration of the antenna.

For both the electro magnetic and acoustic domains, these passive instruments use sensors that cover a very wide spectrum. They have the advantage of being discreet, unlike radar or sonar which emit pulses

\section{Localisation by Conjugated Delays}

* Corresponding author:

pcrumeyrolle@gmail.com (Philippe Crumeyrolle)

Published online at http://journal.sapub.org/ajsp

Copyright (C) 2012 Scientific \& Academic Publishing. All Rights Reserved
Localisation by conjugated delays is a goniometric method based on the conjugation of propagation delays. This method is independent of the application field. When, at an instant $\mathbf{t}_{\text {unknown, an event is triggered by a source }}$ (electromagnetic or acoustic), the wavefront propagates up to the station receivers, which record the signals corresponding to the emitted signal, delayed by the propagation times. A specialised circuit measures the arrival instants of the signal for each antenna sensor with respect to its internal clock. These measurements are processed by the microprocessor that conjugates the delays and determines the direction and range of the signal source. Regardless of whether the field of application is electromagnetic or acoustic, this method requires an antenna of specific geometry

\section{Antenna Configuration}

The antenna is an assembly of base elements, or in short "base". Each base comprises three omnid irectional receiver transducers that are aligned and equally spaced. In other words, a base comprises omnidirectional receivers equipping the ends and centre of a straight-line segment. See figure 1 below.

The antenna is built with bases of length, configuration and number suited for the intended use. Locating a source in a three-dimensional propagation space requires that the antenna be made up of at least three bases that are not aligned and which are not all positioned in the same plane, or of two non-aligned bases and an additional receiver, external to the plane defined by the two bases. The addition of this receiver allows the identification of the part of the space the signal originates from. This elementary antenna is 
static and none of its components requires mechanical or electronic orientation.

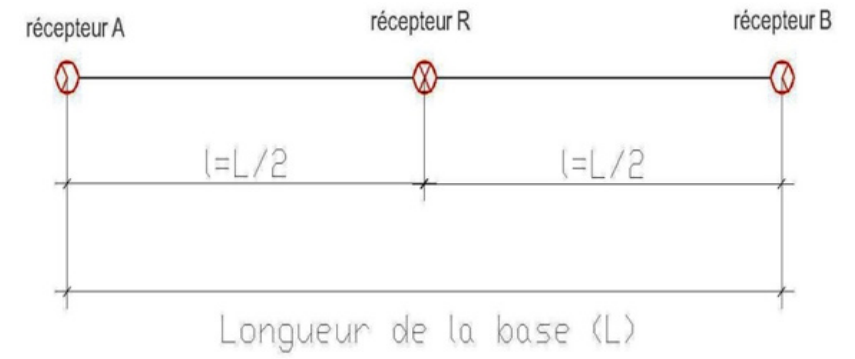

Figure 1. Schema of a base

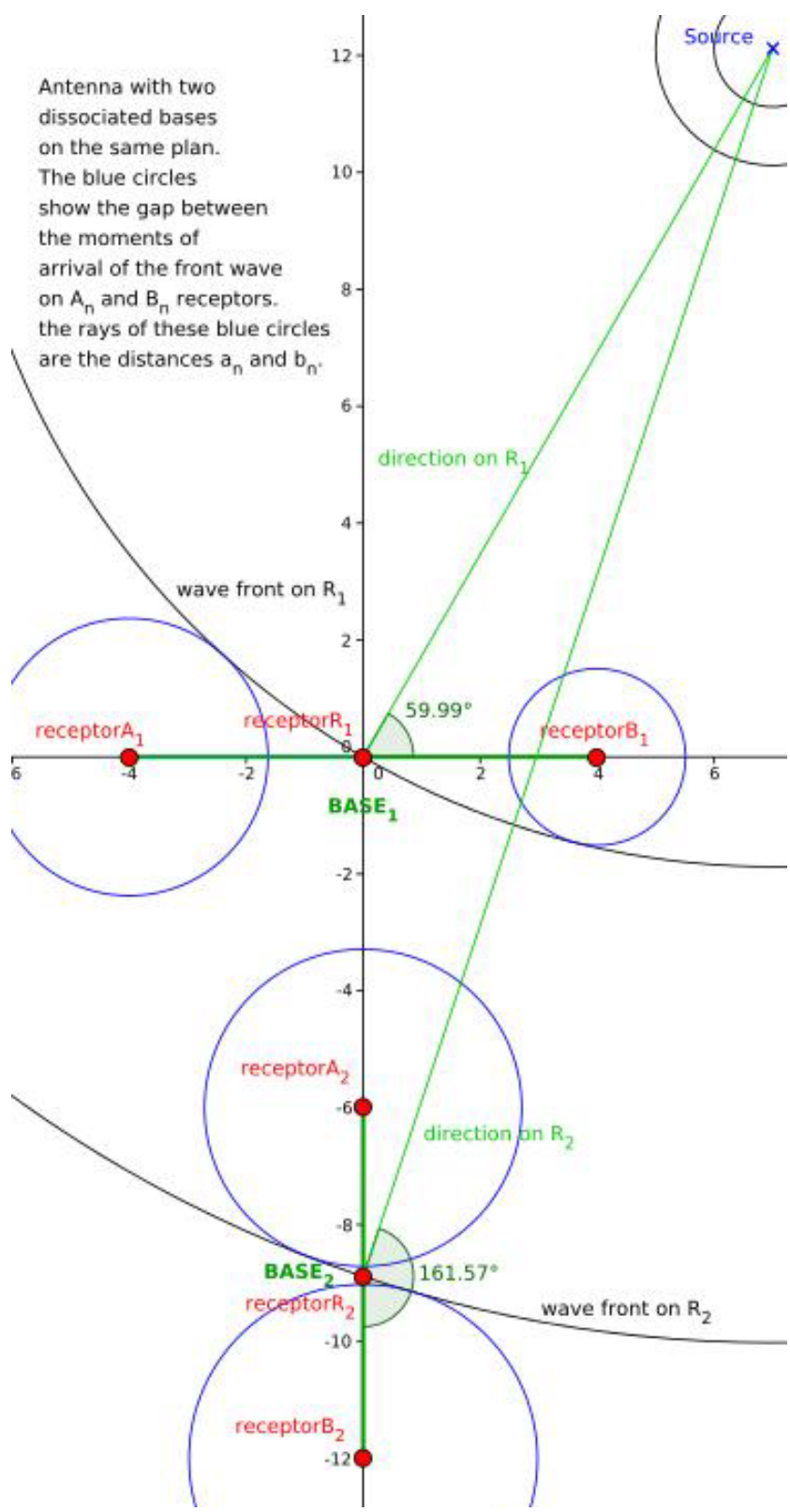

Figure 2. wave front crossing an antenna two bases made

The figure 2 above shows schematically, in the plane formed by the source of the transmitter and antennatwo bases made, a wavefront crossing.

\section{Organisation of Data}

The data used by this method can be classed into the following four main groups.

Construction data: They determine antenna geometry by defining the spacing between the receivers of each base and the configuration of the bases with respect to each other. (Antenna bases may be of different lengths and any configuration).

Assigned data: For each antenna base, three different symbols or letters are assigned to its three receivers and with which an index is associated to distinguish the base. As an illustration, the indexed letters $\mathbf{A}_{\mathrm{n}}$ and $\mathbf{B}_{\mathrm{n}}$ can be assigned to the sensors at the ends of the base, the indexed letter $\mathbf{R}_{\mathrm{n}}$ assigned to a receiver positioned in the centre of base $\mathrm{n}_{\mathrm{n}}$. The distance between receivers $\mathbf{A}_{\mathrm{n}}$ and $\mathbf{R}_{\mathrm{n}}$ is equal to the distance between the receivers $\mathbf{R}_{\mathrm{n}}$ and $\mathbf{B}_{\mathrm{n}}$, with this length between two base receivers called $\boldsymbol{l}_{\mathrm{n}}$.

Physical data: This description of principle does not take account of the physical variables required for a practical application, keeping only signal propagation speed. Here, the propagation speed is considered constant. Signal speed is represented by $\boldsymbol{v}$.

Acquisition data: once the signal of the source to be located has arrived at each antenna receiver, the station's acquisition system produces a report for the arrival times on the receivers $\mathbf{A}_{\mathrm{n}}, \mathbf{R}_{\mathrm{n}}$ and $\mathbf{B}_{\mathrm{n}}$ for each base. These arrival times are called: $\mathrm{DA}_{\mathrm{n}}$ on $\mathbf{A}_{\mathrm{n}}, \mathrm{DR}_{\mathrm{n}}$ on $\mathbf{R}_{\mathrm{n}}, \mathrm{D} \mathbf{B}_{\mathrm{n}}$ on $\mathbf{B}_{\mathrm{n}}$.

The table below shows acollection of TOA sampled from acoustic or electro magnetic source

\section{Method}

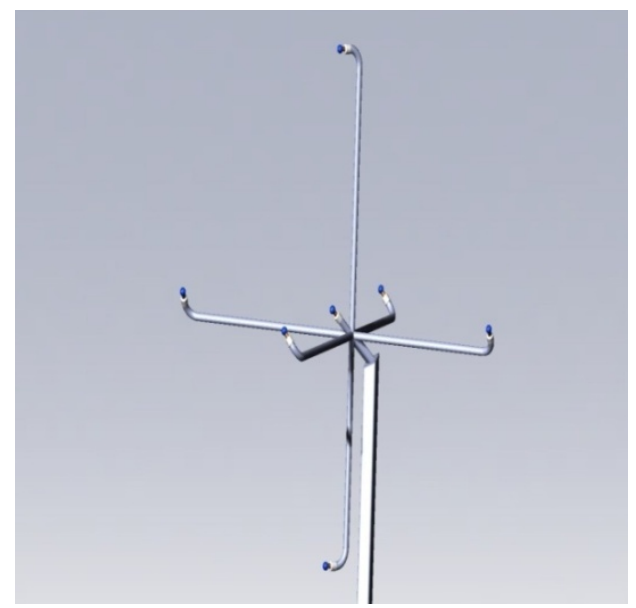

Figure 3. Illustration: Antenna of acoustic direction finder. This antenna made with three bases, their central sensor is common

The principle of the conjugated delays method is simple, the first step consisting of measuring, independently the instant of emission, the signal arrival time on each antenna sensor with respect to the system's temporal reference. The other information (antenna geometry and propagation speed) is already known. 
Table 1. Examples of samples

\begin{tabular}{|c|c|c|c|c|c|}
\hline & \multicolumn{5}{|c|}{ Samples } \\
\hline & signal speed(m/s) & Base $/ 2(\mathrm{~m})$ & \multicolumn{3}{|c|}{ Time of Arrival on receptors } \\
\hline $\mathrm{n}^{\circ}$ & $\mathbf{v}$ & 1 & TOA: $\mathbf{A}$ & TOA: $\mathbf{R}$ & TOA: B \\
\hline 1 & 341 & 0,1 & $8,300010 \mathrm{E}-05$ & $0,000000 \mathrm{E}+00$ & $-8,249270 \mathrm{E}-05$ \\
\hline 2 & 1435 & 3 & $-8,428590 \mathrm{E}-04$ & $1,161432 \mathrm{E}-03$ & $3,167640 \mathrm{E}-03$ \\
\hline 3 & 1500 & 10 & $9,356374 \mathrm{E}-03$ & $3,031250 \mathrm{E}-03$ & $-3,292735 \mathrm{E}-03$ \\
\hline 4 & $3,00 \mathrm{E}+08$ & 20 & $9,169354 \mathrm{E}-08$ & $4,019730 \mathrm{E}-08$ & $-1,128975 \mathrm{E}-08$ \\
\hline
\end{tabular}

The second step is the calculation procedure, operated identically for each of the bases making up the antenna. To generalise, in the following section we consider only one base equipped with the receivers $\mathbf{A}, \mathbf{R}, \mathbf{B}$. The length $\boldsymbol{l}$, is the distance between $\mathbf{A}$ and $\mathbf{R}$ or $\mathbf{R}$ and $\mathbf{B}$, it therefore corresponds to the half-length of the base. This base receives the signal emitted, at an instant $\boldsymbol{t}_{\text {unknown, by the }}$ remote source to be located. The difference in separations between the signal source and the base receivers $\mathbf{A}, \mathbf{R}$ and $\mathbf{B}$, result in differences in the arrival times DA, DR and DB for the wave acquired on these receivers. The signal propagates at the speed $\boldsymbol{v}$, and the distance covered by the wave between the moment DR and the moment DA can be deduced. This distance is $\boldsymbol{a}=\boldsymbol{v} *(\mathrm{DA}-\mathrm{DR})$. In the same way, the distance covered by the wave between the moment DR and the moment DB can also be deducted. This distance is $\boldsymbol{b}=\boldsymbol{v} *(\mathrm{DB}-\mathrm{DR})$. Depending on the emission source, the signal may reach base extremity receivers before reaching the central receiver, and given that the arrival time at receiver $\mathbf{R}$ is the reference time, then measurement of the distance covered is a negative value. We may note that, logically, regardless of the position of the source, the absolute values of $\boldsymbol{a}$ and $\boldsymbol{b}$ remain lower than or equal to the value of $l$.

By conjugating the delay distance $\boldsymbol{a}$, delay distance $\boldsymbol{b}$ and length $\boldsymbol{l}$ via the expression below (1), we obtain the cosine of the angle $\alpha$ between the emission direction and the axis of this base at the point occupied by the receiver $\mathbf{R}$, in the plane formed by this base and the signal source.

$$
\cos (\alpha)=\frac{a^{2} \times b-l^{2} \times b+l^{2} \times a-b^{2} \times a}{l \times\left(-a^{2}+2 \times l^{2}-b^{2}\right)}
$$

*(1)The demonstration of this formula is not the purpose of this note.

This process is applied to each base and the knowledge of antenna geometry and the cosine of each arrival angle leads to the determination of the position of the source using well-known triangulation methods.

In practice, the performance of this method is mainly dependent on the precision of timing of the arrival times of the wave at the antenna receivers and various environmental phenomena such as indexvariation, reflections, etc.

\section{Conclusions}

The passive detection and localization has gained much importance for both military and civilian applications. It has several advantages over active methods, which make it a very viable alternative and sometimes indispensable. The techniques currently in use implements following principles.The radiogoniometry of amplitude using directly the diagram of radiation of the antenna.The radiogoniometry Watson-Watt or with antennas Adcock.The radiogoniometry Doppler, obtained by rotation of an omnidirectional antenna around a center with a rotation speed $\Omega$.The radiogoniometry by interferometry. The radiogoniometry of amplitude and phase based on the high-resolution vectorial corre lation or not.

While, the passive acoustic localization systems can be subcategorized into two groups. Those using the indirect method determine position by a step of estimating time delays between pairs of sensors (TDOA, time differences of arrival) and then step that determines the position based on these delays. Those using the direct method determine the position of the source in one step, by various techniques of beamforming. The main difference with existing techniques is that LCD method, can universally, be implemented in the field of electromagnetic or acoustic applications. This new principle responds to the needs of applications that are based on the capacity of systems to deliver location information instantly and precisely. Regardless of the domain and environment of use, the simplicity of the antenna and the processing resources allow the construction of robust systems that provide determining advantages both in terms of technology and cost.

\section{REFERENCES}

[1] Alon Amar, and Geert Leus, A REFERENCE-FREE TIME DIFFERENCE OF ARRIVAL SOURCE LOCALIZATION USING A PASSIVE SENSOR ARRAY, Faculty of Electrical Engineering, Mathematics and Computer Science, Delft University of Technology, Delft, 2628 CD, The Netherlands, 2010 IEEE Sensor Array and multichannel Signal processing Workshop. 
[2] Jan NEERING, Optimization and Estimation Techniques for Passive Acoustic Source Localization, These de Doctorat, ECOLENATIONALESUPERIEURE DES MINES DE PARIS, avril 2009.

[3] Dinh Thang VU, Alexandre RENAUX, Remy BOYER, Sylvie MARCOS, Analyse des performances de réseaux de capteurs $2 \mathrm{D}$ et $3 \mathrm{D}$ pour la local isation de source, Laboratoire des Signaux et Systèmes (L2S), Université'e Paris-Sud XI, CNRS, SUPELEC, 3, rue Joliot Curie 91192 Gif-sur-Yvette Cedex, France, Manuscrit auteur, publié dans Colloque GRETSI 2009, Dijon : France (2009)

[4] Victor Pratap Singh, Passive Acoustic Localisation (prototype Design an Implementation) For Military Applications, Thesis report for the Degree o Master Of Tehcnology, Department of Computer Science and Engineering Indian Institute of Technology Kanpur, June 2008.

[5] Anthony BELLION, Etude de nouveau concept de systèmes antennaire de radiogoniométrie en polarisation $<<\mathrm{H}>>$ et $<<\mathrm{V}\rangle>$ dans les bandes $<<$ VHF $>>$ et $<<\mathrm{UHF}>>$, Thèse doctorat, Université de limoges, Faculté des sciences et techniques, laboratoire XLIM Départements OSA et $\mathrm{C}^{2} \mathrm{~S}^{2}$ UMR CNRS 6172 Octobre 2008.

[6] J.-L. COLLETTE et O. PIETQUIN, LOCALISATION DE SOURCE SONORE PAR GONIOMETRIE ACOUSTIQUE POUR LA DETECTION DE CHUTE. Supélec - Campus de METZ - 2 rue EdouardBelin - 57070 METZ.

[7] Yushi ZHANG and Waleed H. ABDULLA, A Comparative Study of Time-Delay Estimation Techniques Using Microphone Arrays, School of Engineering Report No. 619, Department of Electrical and Computer Engineering, The University of Auckland, Private Bag 92019, Auckland, New Zealand, 2005

Real-Time Passive Source Localization: A Practical, Linear-Correction Least-Squares Approach, Yiteng Huang, Jacob Benesty, Member, IEEE, Gary W. Elko, and Russell M. Mersereau, Fellow, IEEE, IEEE TRANSACTIONS ON SPEECH AND AUDIO PROCESSING, VOL. 9, NO. 8, NOVEMBER 2001. 